\title{
A Method for the Numerical Integration of Coupled First-Order Differential Equations with Greatly Different Time Constants
}

\section{By Charles E. Treanor}

\begin{abstract}
Coupled differential equations which describe the simultaneous relaxation of different components at greatly different rates present a difficulty in numerical integration, since the integration interval is determined by the fastest rate, and the region of integration is determined by the slowest rate. In the present paper an integration formula is derived from the approximation that within an interval the first derivative can be expressed as $d y / d x=-P y+Q(x)$. The method is exact if the differential equation is of the form shown, where $P$ is constant and $Q(x)$ is a quadratic in $x$. The algorithm utilizes only the first derivative and thus has a parallel to the Runge-Kutta method. For $P h$ small (where $h$ is the integration interval) the method is identical to fourth-order Runge-Kutta and thus is correct to order $h^{4}$. Results for the coupled chemistry of high-temperature air are compared with results obtained from the usual Runge-Kutta procedure.
\end{abstract}

1. Introduction. Of the many methods available for the numerical solution of ordinary differential equations or sets of coupled ordinary differential equations [1], [2] most have specific advantages so that they are chosen for special types of problems. Several methods have a general applicability that has made them popular in a wide variety of cases. There exists, however, a class of equations which present considerable difficulty in their solution and for which there does not seem to be a simple and accurate method available. These equations can be characterized by the fact that the derivative of a dependent variable has a strong dependence on the difference between its own value and that of a slowly varying function. Because of this strong dependence they are sometimes referred to as "stiff" equations [3], [4]. An example is the simultaneous relaxation of several coupled components with widely different relaxation times, where the calculation interval for the entire relaxation region is determined by the time constant of the most rapid process.

In problems of high-temperature air flows, the chemical rate equations sometimes have this character, involving both fast and slow chemical rates in some regions of integration. Similar problems appear in the calculation of both vibrational relaxation and electronic excitation during the dissociation of diatomic molecules. It is desirable to have a method of integration which can handle the stiff-equation problem when it arises, but which will provide the proper speed and accuracy for those portions of the calculation where no special treatment is required.

In the present report a method of integration is derived for the specific purpose of handling this problem. In a case where the derivative is strongly dependent on the value of the dependent variable, the integration formula takes this dependence into account directly and does away with the strong oscillations generated by usual integration formulas such as Runge-Kutta's. However, in the case where the derivative does not have this strong dependence, the method becomes identical

Received March 16, 1965. 
with the fourth-order Runge-Kutta scheme, which is known to handle such integrations with good accuracy. Thus the method can be used for a group of simultaneous differential equations over the entire range of integration when it is not known at what point within the range and for which of the variables the difficulty will occur. A specific application of the method to an aerodynamic chemical-kinetic problem is given as an illustration of the method.

2. Derivation of Integration Formula. The integration formula used for the integration of the first-order differential equation

$$
\frac{d y}{d x}=f(x, y)
$$

is determined by the approximate treatment that is accorded $y(x)$ or $f(x, y)$. An especially convenient method is the fourth-order Runge-Kutta, where $y(x)$ is expanded in a Taylor series, retaining terms to fourth order in the integration interval, $h$. The higher-order derivatives are determined indirectly by evaluating $f(x, y)$ at three collocation points in addition to the initial point. In the usual method [1], these points are given by

$$
\begin{aligned}
x_{2} & =x_{1}+\frac{h}{2}, & y_{2} & =y_{1}+\frac{h}{2} f_{1}, \\
x_{3} & =x_{1}+\frac{h}{2}, & y_{3} & =y_{1}+\frac{h}{2} f_{2}, \\
x_{4} & =x_{1}+h, & y_{4} & =y_{1}+h f_{3},
\end{aligned}
$$

where $y_{i} \equiv y\left(x_{i}\right)$ and $f_{i} \equiv f\left(x_{i}, y_{i}\right)$. Thus $f$ is evaluated at two points midway in the interval and one at each end of the interval. The change in $y$ over the interval is then

$$
(\Delta y)_{\mathbf{R} \cdot \mathbf{K} .}=\frac{h}{6}\left(f_{1}+2 f_{2}+2 f_{3}+f_{4}\right) .
$$

This method of integration, although generally very satisfactory, fails badly in the case where the higher-order derivatives are large [3]. In particular, if Eq. (1) can be written approximately as

$$
\frac{d y}{d x}=-P(y-\tilde{y})
$$

where $P$ is a large number and $\tilde{y}$ is a function of $x$, a difficulty arises because each derivative is $P$ times larger than the preceding one, and so the interval $h$ must be restricted by a condition that $P h$ is not much greater than unity. If $\tilde{y}$ changes only a small fraction of its total range over this interval, then many steps are required to complete the integration.

An alternative procedure is to express $\tilde{y}$ as a power series in $x$ and integrate Eq. (4) directly. An algorithm based on this procedure has been given by Certaine [1], where explicit functions for $P$ and $\tilde{y}$ are utilized in the solution. If, however, the equation to be integrated has a very general form which behaves, in some regions, like that described in Eq. (1), it is convenient to have an integration procedure 
similar to the Runge-Kutta method given in Eqs. (2) and (3). This method requires only the evaluation of $f(x, y)$ at specific points in the integration interval. The present discussion presents a method which satisfies this condition and which, for $P h$ small, is identical with the Runge-Kutta method.

If it is assumed that Eq. (1) can be approximated by

$$
\frac{d y}{d x}=f(x, y)=-P\left(y-y_{1}\right)+A+B\left(x-x_{1}\right)+\frac{1}{2} C\left(x-x_{1}\right)^{2}
$$

over the interval from $x_{1}, y_{1}$, to $x_{1}+h, y_{1}+\Delta y$, then Eq. (5) can be integrated and the value of $\Delta y$ can be written

$$
\Delta y=h\left\{A F_{1}+B h F_{2}+C h^{2} F_{3}\right\} .
$$

The functions $F_{n}$ are simple exponential functions of $P h$

$$
F_{0}=e^{-P h} ; \quad F_{n}=\frac{F_{n-1}-\frac{1}{(n-1) !}}{(-P h)}=\sum_{k=0}^{\infty} \frac{(-P h)^{k}}{(n+k) !} .
$$

The four constants $A, B, C$ and $P$ can be evaluated by determining the value of $f(x, y)$ at four points $x_{i}, y_{i}$ in the interval and solving for the constants from evaluation of Eq. (5) at the four points. The points that are chosen are $\left(x_{1}, y_{1}\right)$ at the beginning of the interval (a point on the true curve), two points at the half interval $x_{2}=x_{3}=x_{1}+h / 2$ and one point at the end of the interval, $x_{4}=x_{1}+h$. The values of $y_{i}$ at the points $x_{i}$ need not be specified as yet. There then results

$$
\begin{aligned}
P & =-\left(\frac{f_{3}-f_{2}}{y_{3}-y_{2}}\right) ; \quad A=f_{1}, \\
B h & =\left[-3\left(f_{1}+P y_{1}\right)+2\left(f_{2}+P y_{2}\right)+2\left(f_{3}+P y_{3}\right)-\left(f_{4}+P y_{4}\right)\right], \\
C h^{2} & =4\left[\left(f_{1}+P y_{1}\right)-\left(f_{2}+P y_{2}\right)-\left(f_{3}+P y_{3}\right)+\left(f_{4}+P y_{4}\right)\right],
\end{aligned}
$$

where $f_{i}=f\left(x_{i}, y_{i}\right)$. Equations (6), (7) and (8) then constitute the integration formula

$$
\begin{aligned}
\Delta y=h\left\{f_{1} F_{1}+\right. & {\left[-3\left(f_{1}+P y_{1}\right)+2\left(f_{2}+P y_{2}\right)+2\left(f_{3}+P y_{3}\right)-\left(f_{4}+P y_{4}\right)\right] F_{2} } \\
+ & \left.4\left[\left(f_{1}+P y_{1}\right)-\left(f_{2}+P y_{2}\right)-\left(f_{3}+P y_{3}\right)+\left(f_{4}+P y_{4}\right)\right] F_{3}\right\}
\end{aligned}
$$

where

$$
P=-\left(\frac{f_{3}-f_{2}}{y_{3}-y_{2}}\right)
$$

It is seen that in the limit of $P \rightarrow 0$ (no dependence of $f(x, y)$ on $y$ ) Eq. (9) becomes equal to the Runge-Kutta formula, Eq. (3), as would be required. If terms of order $h(P h)$ are retained in (9) the result is still identical with (3) providing that the values of $y_{2}, y_{3}$ and $y_{4}$ are chosen according to Eqs. (2). In general, with the definitions for $y_{2}, y_{3}$ and $y_{4}$ given in Eqs. (2), Eq. (9) can be rewritten as

$$
\begin{aligned}
& \Delta y=(\Delta y)_{\mathbf{R} . \mathbf{K}} . \\
& -h(P h)^{2}\left[\left(f_{2}-f_{3}\right) F_{3}+\left(f_{1}-4 f_{2}+2 f_{3}+f_{4}\right) F_{4}-4\left(f_{1}-f_{2}-f_{3}+f_{4}\right) F_{5}\right]
\end{aligned}
$$

where $(\Delta y)_{\mathbf{R . K}}$, is defined in Eq. (3). The additional term is fifth order and higher in $h$. 
Thus when Eq. (9) is used with Eqs. (2) to integrate over an interval where $P h$ is small, the result will be identical with Runge-Kutta. If $P h$ is large, a condition where the Runge-Kutta method is known to be unstable, Eq. (9) supplies a far superior solution.

It should be pointed out that Eq. (10) should not be used for the calculation of $\Delta y$, since when $P$ is large, $(\Delta y)_{\mathbf{R} . \mathbf{k}}$. and the second term in Eq. (10) are each large numbers, while their difference, $\Delta y$, is small. Thus calculation of $\Delta y$ by means of Eq. (10) would be a poor numerical procedure. Equation (10) is included here only to show the close relation between Eq. (9) and the Runge-Kutta formula, and to demonstrate that the error is always of higher order than that obtained with the Runge-Kutta method.

When $P h$ is large it is generally true that the Runge-Kutta equation for $y_{4}$ as given in Eqs. (2) is considerably different from the correct value on the curve, and thus does not provide the best evaluation of the constants $B$ and $C$. Since $P$ is determined from points 2 and $3, F_{j}$ can be evaluated before point 4 is calculated. It is then possible to get a much better approximation for $y_{4}$ by integrating Eq. (5) with the quadratic term omitted, so that

$$
y_{4}=y_{1}+h\left\{2 f_{3} F_{2}+f_{1}\left(F_{1}-2 F_{2}\right)+f_{2}(P h) F_{2}\right\} \text {. }
$$

The use of this equation in place of the last of Eqs. (2) disturbs the result for $\Delta y$ from the Runge-Kutta answer only to fifth order in $h$ and is very helpful when $P h$ is large.

In the limit of $P h$ very large, Eq. (9) becomes

$$
\lim _{P h \rightarrow \infty} \Delta y \rightarrow=\left(\frac{f_{4}+P y_{4}}{P}\right)-y_{1}=\tilde{y}_{4}-y_{1} .
$$

Thus $y$ approaches the equilibrium value $\tilde{y}$ evaluated at $x=x_{1}+h$. This limiting solution is obtained independent of the relations chosen between $y_{i}$ and $x_{i}$.

A numerical problem in the application of Eq. (9) can arise in the evaluation of $P$. Since this evaluation involves the difference of two values of $f_{i}$, it is possible to lose many of the significant figures in the $P$ calculation if the change in $f_{i}$ is very small, and it is even possible to obtain a negative value for $P$. Thus as a practical consideration the sign of $P$ should be tested and if it is negative it should be set equal to zero, so that Eq. (9) reverts to the Runge-Kutta method.

3. Example. The example that has been chosen to illustrate the advantages of the present integration procedure is taken from the coupled chemical-kinetic equations for reactions behind a shock wave in air. A discussion of the reactions and a complete description of the coupled equations can be found in [5]. The coupled equations describe the complicated time-history of the air behind a strong shock wave where the high-temperature oxygen and nitrogen react chemically to form nitric oxide, oxygen and nitrogen atoms, and various atomic and molecular ions. The dependent variables are these species concentrations and the enthalpy and velocity of the air. The independent variable, $x$, is the distance behind the shock wave and thus is proportional to the time since the reactions started. In the example used here the temperature immediately behind the shock wave is $3070^{\circ} \mathrm{K}$ and the density is $8.18 \times 10^{-3} \mathrm{gms} / \mathrm{cm}^{3}$. Six atomic and molecular species are con- 


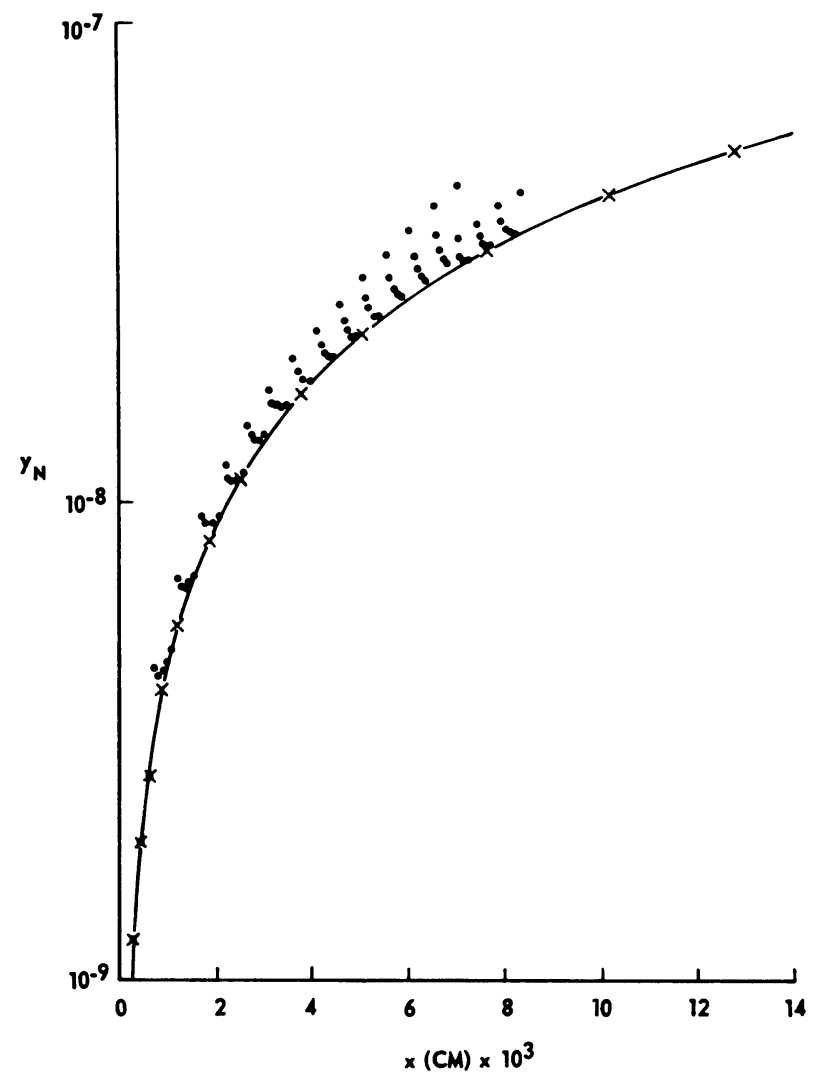

Figure 1. Nitrogen atom concentration behind shock in air. $V_{t}=2.60 \mathrm{~mm} / \mu \mathrm{sec} ; P_{1}$ $=1 \mathrm{~atm}$. Runge-Kutta, $X$ present method.

sidered, and nine chemical reactions. The temperature is sufficiently low so that ionization can be neglected.

The differential equation for the rate of formation of nitrogen atoms can be written

$$
\begin{aligned}
\frac{d y_{\mathrm{N}}}{d x}= & D_{1} y_{\mathrm{N}_{2}} y_{\mathrm{M}}-D_{1}^{\prime} y_{\mathrm{N}}^{2} y_{\mathrm{M}}+D_{2} y_{\mathrm{No}} y_{\mathrm{M}}-D_{2}^{\prime} y_{\mathrm{N}} y_{\mathrm{o}} y_{\mathrm{M}}-D_{3} y_{\mathrm{N}} y_{\mathrm{O}_{2}} \\
& +D_{3}^{\prime} y_{\mathrm{No}} y_{\mathrm{o}}+D_{4} y_{\mathrm{o}} y_{\mathrm{N}_{2}}-D_{4}^{\prime} y_{\mathrm{No}} y_{\mathrm{N}}
\end{aligned}
$$

where the coefficients $D_{i}$ are functions of the over-all density and temperature of the air. The dependent variables are the species concentrations, identified by the various letter subscripts. There are similar differential equations for each of these species. During much of the flow regime the terms $-D_{3} y_{\mathrm{N}} y_{\mathrm{O}_{2}}$ and $D_{3}{ }^{\prime} y_{\mathrm{NO}} y_{\mathrm{O}}$ are considerably larger than the other terms, and thus control the differential equation. These are the "short time-constant" terms which are emphasized in the form of Eq. (4).

The results obtained for the nitrogen atom concentrations, using the fourth-order Runge-Kutta scheme of integration is shown by the points in Figure 1. After completion of each interval of integration a number of tests are performed, and failure 
of any of these tests results in discarding the last step of integration and cutting the integration interval by a factor of two [6]. In addition, after two successful steps, the integration interval is doubled, in an attempt to proceed at the largest possible integration interval.

The Runge-Kutta results shown in Figure 1 show that a sharp saw-tooth rise results whenever the interval is doubled from $0.8 \times 10^{-4} \mathrm{~cm}$ to $1.6 \times 10^{-4} \mathrm{~cm}$. This resulting calculation is in error, but not enough to fail the integration tests. The next step at the larger interval does fail the tests and reduces the interval again. At this interval the calculated points approach the correct curve. After two successful steps the interval is doubled, but the result fails the tests, and so does not appear in Figure 1. After two more steps the calculated values are very close to the correct curve, and when the interval is doubled the result, though poor, does pass the tests imposed. In this manner the integration continues the saw-tooth pattern.

The method presented in the present paper was also applied to this problem with the same rules for testing integration accuracy and obtaining interval size. Equations (2) were used for $y_{2}$ and $y_{3}$, and Eq. (11) was used for $y_{4}$. Equation (9) was then used to obtain $\Delta y$. It should be emphasized that the calculation time per integration step is essentially the same as for the Runge-Kutta method, since most of the computation time is spent in evaluating the derivatives, so that the extra time spent in evaluating Eq. (9) instead of (3) is negligible. The rasults are shown by the $x$ 's in Figure 1. It is seen that the interval increases to $2.56 \times 10^{-3} \mathrm{~cm}$, some twenty-five times larger than the Runge-Kutta step. The value of $P h$ for these steps is $\sim 75$. As shown in [3], the fourth-order Runge-Kutta equations are not. stable for this form of equation for $P h>5.6$. This is consistent with the present numerical results, where a Runge-Kutta step of $1 / 25$ of that of the present method $(P h \sim 3)$ is stable, but twice that step size $(P h \sim 6)$ is not.

The nitrogen atom concentration is shown for comparison in Figure 1 because it is this species which provides the numerical difficulty in the present problem. All the other species concentrations follow smooth curves throughout the integration.

Acknowledgment. The research described in this paper has been sponsored by the National Aeronautics and Space Administration under Contract No. NASr-119. The author would like to thank Mr. Leonard Garr, who programmed these calculations, for several helpful discussions of the numerical procedures. In addition he would like to express appreciation to Miss Marcia Williams for assistance in analysis of the results. For introduction to this problem and for many subsequent discussions, he is indebted to Mr. Paul Marrone.

Cornell Aeronautical Laboratory, Inc.

Buffalo, New York

1. A. Ralston \& H.S. Wilf (Eds.), Mathematical Methods for Digital Computers, Wiley, New York, 1960. MR 22 *8680.

2. S. H. Crandald, Engineering Analysis; A Survey of Numerical Procedures, McGrawHill, New York, 1956. MR 18, \#674.

3. G. EManuel, Problems Underlying the Numerical Integration of the Chemical and Vibrational Rate Equations in a Near Equilibrium Flow, Report AEDC-TDR-63-82, Arnold Engineering Development Center, Tullahoma, Tenn., 1963. 
4. C. F. Curtiss \& J. O. Hirschfelder, "Integration of stiff equations," Proc. Nat. Acad. Sci. U.S.A., v. 38, 1952, pp. 235-243. MR 13, *873.

5. P. V. MARrone, Inviscid, Nonequilibrium Flow Behind Bow and Normal Shock Waves, Part I. General Analysis and Numerical Examples, Report QM-1626-A-12(I), Cornell Aeronautical Laboratory, Buffalo, N.Y., 1963.

6. P. V. Marrone \& L. J. GarR, Inviscid, Nonequilibrium Flow Behind Bow and Normal Shock Waves, Part II. The IBM 704 Computer Programs, Report QM-1626-A-12(II), Cornell Aeronautical Laboratory, Buffalo, N.Y., 1963. 